\title{
ALMOST SURE AND MOMENT EXPONENTIAL STABILITY IN THE NUMERICAL SIMULATION OF STOCHASTIC DIFFERENTIAL EQUATIONS*
}

\author{
DESMOND J. HIGHAM ${ }^{\dagger}$, XUERONG MAO M AND CHENGGUI YUAN $^{\S}$
}

\begin{abstract}
Relatively little is known about the ability of numerical methods for stochastic differential equations (SDEs) to reproduce almost sure and small-moment stability. Here, we focus on these stability properties in the limit as the timestep tends to zero. Our analysis is motivated by an example of an exponentially almost surely stable nonlinear SDE for which the Euler-Maruyama (EM) method fails to reproduce this behavior for any nonzero timestep. We begin by showing that EM correctly reproduces almost sure and small-moment exponential stability for sufficiently small timesteps on scalar linear SDEs. We then generalize our results to multidimensional nonlinear SDEs. We show that when the SDE obeys a linear growth condition, EM recovers almost surely exponential stability very well. Under the less restrictive condition that the drift coefficient of the SDE obeys a one-sided Lipschitz condition, where EM may break down, we show that the backward Euler method maintains almost surely exponential stability.
\end{abstract}

Key words. backward Euler, Euler-Maruyama, implicit, one-sided Lipschitz condition, linear growth condition, Lyapunov exponent, stochastic theta method

AMS subject classifications. $65 \mathrm{C} 30,60 \mathrm{H} 10$

DOI. $10.1137 / 060658138$

1. Introduction. Stability theory for numerical simulations of stochastic differential equations (SDEs) typically deals with mean-square behavior. Asymptotic, or almost sure, stability is at least as relevant in typical applications, but does not benefit from a well-developed theory. Our general aim here is to address this imbalance.

We begin with a brief overview of relevant work.

A characterization of asymptotic linear stability for a wide class of SDE methods was given in [10, Lemma 5.1], but this turns out to be of limited use in proving analytical results. Some properties for weak methods were derived in [10, section 6], and results for the related $T$-stability concept can be found in [19]. The issue of whether the asymptotic linear stability region is bounded was analyzed in [5]. Other authors $[7,8,17]$ have tested asymptotic stability via numerical experiments.

The related concept of $p$ th moment stability for $0<p \leq 2$ is interesting in its own right, and in the linear scalar SDE case it is known that as $p \rightarrow 0$ this property is equivalent to asymptotic stability; see Theorem 4.1. We note that some analysis in [1] on stochastic difference equations is relevant to the application of a weak Euler-Maruyama (EM) method to a scalar SDE, and further strengthens the connection between $p$ th moment and asymptotic stability. Similarly, the results in [2] are relevant to EM on a scalar SDE; in this case the emphasis is on polynomial, rather

\footnotetext{
*Received by the editors April 25, 2006; accepted for publication (in revised form) October 2, 2006; published electronically April 3, 2007.

http://www.siam.org/journals/sinum/45-2/65813.html

$\dagger$ Department of Mathematics, University of Strathclyde, Glasgow, G1 1XH, UK (djh@maths. strath.ac.uk).

${ }^{\ddagger}$ Department of Statistics and Modelling Science, University of Strathclyde, Glasgow G1 1XH, UK (xuerong@stams.strath.ac.uk).

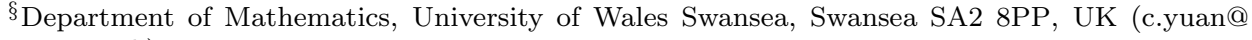
swansea.ac.uk).
} 
than the generic exponential, rates of convergence. In [4], moment stability for SDEs with delay is studied in the presence of a suitable Lyapunov function.

Unlike in the mean-square case [10], we are not aware of any numerical methods that, on a reasonable class of SDEs, have been proved to possess an asymptotic stability analogue of deterministic A-stability [9]; "problem stable implies numerical method stable for all stepsizes."

In this work, we focus on a more fundamental property of the form "problem stable implies numerical method stable for sufficiently small stepsizes," where stability is meant in the exponential asymptotic sense and independently of the size of initial data. To show that this is a nontrivial issue, we give a nonlinear example in section 3 where, for arbitrarily small timesteps, the basic EM method may fail to preserve stability. This motivates the subsequent analysis. We find conditions under which EM does preserve exponential asymptotic stability for small timesteps, and we show that introducing implicitness, in the form of the backward Euler method, produces good results on a class of SDEs that includes our motivating example.

More precisely, we prove positive results for scalar-noise SDEs that are linear (section 4) or satisfy linear growth conditions (section 5). Then in section 6 we show that backward Euler is successful under a one-sided Lipschitz condition on the drift. Section 7 shows how the results generalize to multidimensional noise.

2. Notation. Throughout this paper, we let $\left(\Omega, \mathcal{F},\left\{\mathcal{F}_{t}\right\}_{t \geq 0}, \mathbb{P}\right)$ be a complete probability space with a filtration $\left\{\mathcal{F}_{t}\right\}_{t \geq 0}$ that is increasing and right continuous, with $\mathcal{F}_{0}$ containing all $\mathbb{P}$-null sets. Let $B(t)$ be a scalar Brownian motion defined on the probability space. Let $|\cdot|$ denote both the Euclidean norm in $\mathbb{R}^{n}$ and the trace (or Frobenius) norm in $\mathbb{R}^{n \times m}$. The inner product of $x, y$ in $\mathbb{R}^{n}$ is denoted by $\langle x, y\rangle$. We use $a \vee b$ to denote $\max (a, b), a \wedge b$ to denote $\min (a, b)$, and a.s. to mean almost surely.

We are concerned with the $n$-dimensional nonlinear Itô SDE

$$
d x(t)=f(x(t)) d t+g(x(t)) d B(t), \quad t \geq 0, \quad \text { given } 0 \neq x(0) \in \mathbb{R}^{n} .
$$

As a standing hypothesis, we assume that $f, g: \mathbb{R}^{n} \rightarrow \mathbb{R}^{n}$ are smooth enough for the SDE $(2.1)$ to have a unique global solution $x(t)$ on $[0, \infty)$ (see, for example, [15], for sufficient conditions). We make two remarks.

- Scalar Brownian motion $B(t)$ is used to make the analysis in sections 5 and 6 more accessible. In section 7 we state how our results extend to the case of multidimensional noise.

- The restriction to a deterministic initial condition is convenient and does not lose any generality when asymptotic stability is studied; see, for example, [15, section 4.2].

The EM method applied to (2.1) produces approximations $X_{k} \approx x(k \Delta t)$, where $X_{0}=x(0)$ and

$$
X_{k+1}=X_{k}+\Delta t f\left(X_{k}\right)+g\left(X_{k}\right) \Delta B_{k} .
$$

Here $\Delta t>0$ is the timestep and $\Delta B_{k}:=B((k+1) \Delta t)-B(k \Delta t)$ is the Brownian increment. We will also consider the more general stochastic theta (ST) method which takes the form

$$
X_{k+1}=X_{k}+\Delta t\left((1-\theta) f\left(X_{k}\right)+\theta f\left(X_{k+1}\right)\right)+g\left(X_{k}\right) \Delta B_{k},
$$

where $\theta \in[0,1]$ is a fixed parameter. For $\theta=0$, ST reduces to EM. For $\theta \neq 0(2.3)$ defines $X_{k+1}$ implicitly. We will refer to the $\theta=1$ case as backward Euler (BE). 
3. Motivating example. For the scalar cubic SDE

$$
d x(t)=\left(x(t)-x(t)^{3}\right) d t+2 x(t) d B(t)
$$

it follows from Theorem 6.1 in section 6 below that

$$
\limsup _{t \rightarrow \infty} \frac{1}{t} \log |x(t)| \leq-1 \quad \text { a.s. }
$$

The EM method (2.2) applied to (3.1) produces

$$
X_{k+1}=X_{k}\left(1+\Delta t-\Delta t X_{k}^{2}+2 \Delta B_{k}\right) .
$$

Lemma 3.1. Suppose $0<\Delta t<1$. If $\left|X_{1}\right| \geq 2^{4} / \sqrt{\Delta t}$ in (3.3), then

$$
\mathbb{P}\left(\left|X_{k}\right| \geq \frac{2^{k+3}}{\sqrt{\Delta t}} \forall k \geq 1\right) \geq \exp \left(-4 e^{-2 / \sqrt{\Delta t}}\right) .
$$

Proof. First, we show that

$$
\left|X_{k}\right| \geq \frac{2^{k+3}}{\sqrt{\Delta t}} \text { and }\left|\Delta B_{k}\right| \leq 2^{k} \quad \Rightarrow \quad\left|X_{k+1}\right| \geq \frac{2^{k+4}}{\sqrt{\Delta t}} .
$$

To see this, suppose $\left|X_{k}\right| \geq 2^{k+3} / \sqrt{\Delta t}$. Then

$$
\begin{aligned}
\left|X_{k+1}\right| & \geq\left|X_{k}\right||\Delta t| X_{k}^{2}|-1-\Delta t-2| \Delta B_{k}|| \\
& \geq \frac{2^{k+3}}{\sqrt{\Delta t}}\left|2^{2 k+6}-1-\Delta t-2\right| \Delta B_{k}|| .
\end{aligned}
$$

Hence, $\left|X_{k+1}\right| \geq 2^{k+4} / \sqrt{\Delta t}$ if

$$
2^{2 k+6}-1-\Delta t-2\left|\Delta B_{k}\right| \geq 2
$$

that is,

$$
2\left|\Delta B_{k}\right| \leq 2^{2 k+6}-3-\Delta t .
$$

Since $2^{2 k+6}-3-\Delta t \geq 2^{2 k+6}-4 \geq 2^{k+1} \forall k \geq 0$, the implication (3.4) follows.

From (3.4), given that $\left|X_{1}\right| \geq 2^{4} / \sqrt{\Delta t}$, the event that $\left\{\left|X_{k}\right| \geq 2^{k+3} / \sqrt{\Delta t}, \forall 1 \leq\right.$ $k \leq K\}$ contains the event that $\left\{\left|\Delta B_{k}\right| \leq 2^{k} \forall 1 \leq k \leq K\right\}$. So, because the $\left\{\Delta B_{k}\right\}$ are independent,

$$
\mathbb{P}\left(\left|X_{k}\right| \geq \frac{2^{k+3}}{\sqrt{\Delta t}} \forall 1 \leq k \leq K\right) \geq \prod_{k=1}^{K} \mathbb{P}\left(\left|\Delta B_{k}\right| \leq 2^{k}\right) .
$$

Now, because $\Delta B_{k} \sim N(0, \Delta t)$, we have

$$
\begin{aligned}
\mathbb{P}\left(\left|\Delta B_{k}\right| \geq 2^{k}\right) & =\mathbb{P}\left(\frac{\left|\Delta B_{k}\right|}{\sqrt{\Delta t}} \geq \frac{2^{k}}{\sqrt{\Delta t}}\right) \\
& =\frac{2}{\sqrt{2 \pi}} \int_{2^{k} / \sqrt{\Delta t}}^{\infty} e^{-x^{2} / 2} d x \\
& \leq \frac{2}{\sqrt{2 \pi}} \int_{2^{k} / \sqrt{\Delta t}}^{\infty} e^{-x} d x \\
& =\frac{2}{\sqrt{2 \pi}} \exp \left(-\frac{2^{k}}{\sqrt{\Delta t}}\right)
\end{aligned}
$$


Hence, in (3.5)

$$
\mathbb{P}\left(\left|X_{k}\right| \geq \frac{2^{k+3}}{\sqrt{\Delta t}} \forall 1 \leq k \leq K\right) \geq \prod_{k=1}^{K}\left(1-\exp \left(-\frac{2^{k}}{\sqrt{\Delta t}}\right)\right) .
$$

Since

$$
\log (1-u) \geq-2 u \quad \text { for } 0<u<\frac{1}{2}
$$

we then have

$$
\begin{aligned}
\log \left(\mathbb{P}\left(\left|X_{k}\right| \geq \frac{2^{k+3}}{\sqrt{\Delta t}} \forall 1 \leq k \leq K\right)\right) & \geq \sum_{k=1}^{K} \log \left(1-\exp \left(-\frac{2^{k}}{\sqrt{\Delta t}}\right)\right) \\
& \geq-2 \sum_{k=1}^{K} \exp \left(-\frac{2^{k}}{\sqrt{\Delta t}}\right) .
\end{aligned}
$$

Next, using $2^{k} \geq 2 k$,

$$
\sum_{k=1}^{K} \exp \left(\frac{-2^{k}}{\sqrt{\Delta t}}\right) \leq \sum_{k=1}^{K} \exp \left(-\frac{2 k}{\sqrt{\Delta t}}\right)
$$

The right-hand side is a geometric series that converges monotonically from below to $e^{-2 / \sqrt{\Delta t}} /\left(1-e^{-2 / \sqrt{\Delta t}}\right) \leq 2 e^{-2 / \sqrt{\Delta t}}$. Hence, in (3.6),

$$
\log \left(\mathbb{P}\left(\left|X_{k}\right| \geq \frac{2^{k+3}}{\sqrt{\Delta t}} \forall 1 \leq k \leq K\right)\right) \geq-4 e^{-2 / \sqrt{\Delta t}},
$$

and the result follows.

To interpret Lemma 3.1, we note that given any $x(0) \neq 0$ and any $\Delta t>0$, there is a nonzero probability that the first Brownian increment, $\Delta B_{1}$, will cause $\left|X_{1}\right| \geq$ $2^{4} / \sqrt{\Delta t}$. Hence, there is a nonzero probability that EM will produce a numerical solution that blows up at a geometric rate. This contrasts with the initial-dataindependent exponential stability of the underlying SDE, shown by (3.2).

In sections 4 and 5 we show that this poor behavior cannot happen when EM is applied to linear scalar problems or an appropriate class of nonlinear SDEs. In section 6 we study a class of SDEs that includes (3.1) and show that the correct stability can be retained by moving to an implicit method. We note that in all results, when we state the existence of a suitable upper limit, $\Delta t^{\star}$, on the stepsize, we implicitly mean that $\Delta t^{\star}$ does not depend on the initial data.

4. Linear scalar SDEs. In this section we focus on the linear scalar SDE

$$
d x(t)=\alpha x(t) d t+\sigma x(t) d B(t), \quad \text { with } 0 \neq x(0) \in \mathbb{R},
$$

where $\alpha$ and $\sigma$ are real numbers. The following result is classical; see, e.g., $[3,13,14]$.

THEOREM 4.1. The sample Lyapunov exponent of the solution to the SDE (4.1) is

$$
\lim _{t \rightarrow \infty} \frac{1}{t} \log |x(t)|=\alpha-\frac{1}{2} \sigma^{2} \quad \text { a.s. }
$$


and the pth moment Lyapunov exponent is

$$
\lim _{t \rightarrow \infty} \frac{1}{t} \log \mathbb{E}\left(|x(t)|^{p}\right)=p \alpha+\frac{1}{2} p(p-1) \sigma^{2},
$$

for any $p>0$. Hence, the zero solution of the SDE (4.1) is a.s. exponentially stable if and only if $\alpha-\frac{1}{2} \sigma^{2}<0$, while it is pth moment exponentially stable if and only if $\alpha+\frac{1}{2}(p-1) \sigma^{2}<0$.

We hence observe that the zero solution of the SDE (4.1) is a.s. exponentially stable if and only if it is $p$ th moment exponentially stable for some sufficiently small positive $p$.

In the following three subsections we show that for small $\Delta t$, EM and ST recover almost sure and $p$ th moment exponential stability of (4.1).

\subsection{Almost sure exponential stability of Euler-Maruyama.}

THEOREM 4.2. If $\alpha-\frac{1}{2} \sigma^{2}<0$ in $(4.1)$, then for any $\varepsilon \in(0,1)$ there is a $\Delta t^{\star} \in(0,1)$ such that for any $\Delta t<\Delta t^{\star}$, the EM approximation has the property that

$$
\lim _{k \rightarrow \infty} \frac{1}{k \Delta t} \log \left|X_{k}\right| \leq(1-\varepsilon)\left(\alpha-\frac{1}{2} \sigma^{2}\right)<0 \quad \text { a.s. }
$$

Proof. The EM method (2.2) applied to (4.1) has the form

$$
X_{k+1}=X_{k}\left(1+\alpha \Delta t+\sigma \Delta B_{k}\right) .
$$

It follows that $X_{k}=x_{0} \prod_{j=0}^{k-1}\left(1+\alpha \Delta t+\sigma \Delta B_{j}\right)$, and thus

$$
\log \left|X_{k}\right|=\log \left|x_{0}\right|+\sum_{j=0}^{k-1} \log \left|1+\alpha \Delta t+\sigma \Delta B_{j}\right| .
$$

Dividing both sides by $k$, letting $k \rightarrow \infty$, and then applying the classical strong law of large numbers we obtain

$$
\lim _{k \rightarrow \infty} \frac{1}{k \Delta t} \log \left|X_{k}\right|=\frac{1}{\Delta t} \mathbb{E} \log |1+\alpha \Delta t+\sigma \sqrt{\Delta} Z| \quad \text { a.s., } \quad \text { where } Z \sim N(0,1) .
$$

Writing

$$
\begin{aligned}
\log |1+\alpha \Delta t+\sigma \sqrt{\Delta t} Z| & =\frac{1}{2} \log \left([1+\alpha \Delta t+\sigma \sqrt{\Delta t} Z]^{2}\right) \\
& =\frac{1}{2} \log \left(1+2[\alpha \Delta t+\sigma \sqrt{\Delta t} Z]+[\alpha \Delta t+\sigma \sqrt{\Delta t} Z]^{2}\right),
\end{aligned}
$$

and recalling the fundamental inequality

$$
\log (1+u) \leq u-\frac{1}{2} u^{2}+\frac{1}{3} u^{3}, \quad u \geq-1,
$$

we have

$$
\begin{aligned}
\log |1+\alpha \Delta t+\sigma \sqrt{\Delta t} Z| \leq & \frac{1}{2}\left(2[\alpha \Delta t+\sigma \sqrt{\Delta t} Z]+[\alpha \Delta t+\sigma \sqrt{\Delta t} Z]^{2}\right. \\
& -\frac{1}{2}\left(2[\alpha \Delta t+\sigma \sqrt{\Delta t} Z]+[\alpha \Delta t+\sigma \sqrt{\Delta t} Z]^{2}\right)^{2} \\
& \left.+\frac{1}{3}\left(2[\alpha \Delta t+\sigma \sqrt{\Delta t} Z]+[\alpha \Delta t+\sigma \sqrt{\Delta t} Z]^{2}\right)^{3}\right)
\end{aligned}
$$


Making use of the properties $\mathbb{E}\left(Z^{2 n}\right)=(2 n-1) ! !$ and $\mathbb{E}\left(Z^{2 n-1}\right)=0$, for $n=1,2,3, \ldots$, we can compute

$$
\left\{\begin{aligned}
\mathbb{E}[\alpha \Delta t+\sigma \sqrt{\Delta t} Z] & =\alpha \Delta t \\
\mathbb{E}\left([\alpha \Delta t+\sigma \sqrt{\Delta t} Z]^{2}\right) & =\alpha^{2} \Delta t^{2}+\sigma^{2} \Delta t \\
\mathbb{E}\left([\alpha \Delta t+\sigma \sqrt{\Delta t} Z]^{3}\right) & =\alpha^{3} \Delta t^{3}+3 \alpha \sigma^{2} \Delta t^{2}, \\
\mathbb{E}\left([\alpha \Delta t+\sigma \sqrt{\Delta t} Z]^{4}\right) & =\alpha^{4} \Delta t^{4}+6 \alpha^{2} \sigma^{2} \Delta t^{3}+3 \sigma^{4} \Delta t^{2}, \\
\mathbb{E}\left([\alpha \Delta t+\sigma \sqrt{\Delta t} Z]^{5}\right) & =\alpha^{5} \Delta t^{5}+10 \alpha^{3} \sigma^{2} \Delta t^{4}+15 \alpha \sigma^{4} \Delta t^{3}, \\
\mathbb{E}\left([\alpha \Delta t+\sigma \sqrt{\Delta t} Z]^{6}\right) & =\alpha^{6} \Delta t^{6}+15 \alpha^{4} \sigma^{2} \Delta t^{5}+45 \alpha^{2} \sigma^{4} \Delta t^{4}+15 \sigma^{6} \Delta t^{3},
\end{aligned}\right.
$$

and hence obtain

$$
\mathbb{E} \log |1+\alpha \Delta t+\sigma \sqrt{\Delta t} Z| \leq\left(\alpha-\frac{1}{2} \sigma^{2}\right) \Delta t+C_{1} \Delta t^{2},
$$

where $C_{1}=C_{1}(\alpha, \sigma)>0$ is a constant independent of $\Delta t$. Now, choose $\Delta t^{\star} \in(0,1)$ so small that $C_{1} \Delta t^{\star} \leq \varepsilon\left(\frac{1}{2} \sigma^{2}-\alpha\right)$. Then for any $\Delta t<\Delta t^{\star}$ we can substitute (4.6) into (4.4) to obtain (4.2).

\subsection{Moment exponential stability of Euler-Maruyama.}

TheOREM 4.3. Let $p \in(0,2]$. If $\alpha+\frac{1}{2}(p-1) \sigma^{2}<0$ in (4.1), then for any $\varepsilon \in(0,1)$ there is a $\Delta t^{\star} \in(0,1)$ such that for any $\Delta t<\Delta t^{\star}$, the EM approximation has the property that

$$
\lim _{k \rightarrow \infty} \frac{1}{k \Delta t} \log \mathbb{E}\left(\left|X_{k}\right|^{p}\right) \leq(1-\varepsilon) p\left(\alpha+\frac{1}{2}(p-1) \sigma^{2}\right)<0 .
$$

Proof. It follows from (4.3) that $\mathbb{E}\left(\left|X_{k}\right|^{p}\right)=\left|x_{0}\right|^{p} \prod_{j=0}^{k-1} \mathbb{E}\left(\left|1+\alpha \Delta t+\sigma \Delta B_{j}\right|^{p}\right)$, and hence

$$
\mathbb{E}\left(\left|X_{k}\right|^{p}\right)=\left|x_{0}\right|^{p}\left(\mathbb{E}|1+\alpha \Delta t+\sigma \sqrt{\Delta} Z|^{p}\right)^{k}, \quad \text { where } Z \sim N(0,1) .
$$

This implies

$$
\lim _{k \rightarrow \infty} \frac{1}{k \Delta t} \log \mathbb{E}\left(\left|X_{k}\right|^{p}\right)=\frac{1}{\Delta t} \log \mathbb{E}\left(|1+\alpha \Delta t+\sigma \sqrt{\Delta} Z|^{p}\right) .
$$

Writing

$$
\begin{aligned}
|1+\alpha \Delta t+\sigma \sqrt{\Delta t} Z|^{p} & =\left([1+\alpha \Delta t+\sigma \sqrt{\Delta t} Z]^{2}\right)^{p / 2} \\
& =\left(1+2[\alpha \Delta t+\sigma \sqrt{\Delta t} Z]+[\alpha \Delta t+\sigma \sqrt{\Delta t} Z]^{2}\right)^{p / 2},
\end{aligned}
$$

and recalling the fundamental inequality

$$
(1+u)^{p / 2} \leq 1+\frac{p}{2} u+\frac{p(p-2)}{8} u^{2}+\frac{p(p-2)(p-4)}{2^{3} \times 3 !} u^{3}, \quad u \geq-1,
$$

we have

$$
\begin{aligned}
|1+\alpha \Delta t+\sigma \sqrt{\Delta t} Z|^{p} \leq & 1+\frac{p}{2}\left(2[\alpha \Delta t+\sigma \sqrt{\Delta t} Z]+[\alpha \Delta t+\sigma \sqrt{\Delta t} Z]^{2}\right) \\
& +\frac{p(p-2)}{8}\left(2[\alpha \Delta t+\sigma \sqrt{\Delta t} Z]+[\alpha \Delta t+\sigma \sqrt{\Delta t} Z]^{2}\right)^{2} \\
& +\frac{p(p-2)(p-4)}{48}\left(2[\alpha \Delta t+\sigma \sqrt{\Delta t} Z]+[\alpha \Delta t+\sigma \sqrt{\Delta t} Z]^{2}\right)^{3} .
\end{aligned}
$$


Making use of (4.5), we obtain

$$
\mathbb{E}\left(|1+\alpha \Delta t+\sigma \sqrt{\Delta t} Z|^{p}\right) \leq 1+p\left[\alpha+\frac{1}{2}(p-1) \sigma^{2}\right] \Delta t+C_{3} \Delta t^{2}
$$

where $C_{3}=C_{3}(\alpha, \sigma, p)>0$ is a constant independent of $\Delta t$. Now, choose $\Delta t^{\star} \in(0,1)$ so small that for all $\Delta t<\Delta t^{\star}$

$$
C_{3} \Delta t \leq \varepsilon p\left|\alpha+\frac{1}{2}(p-1) \sigma^{2}\right| \quad \text { and } \quad-1<(1-\varepsilon) p\left[\alpha+\frac{1}{2}(p-1) \sigma^{2}\right] \Delta t<0 .
$$

Then

$$
\mathbb{E}\left(|1+\alpha \Delta t+\sigma \sqrt{\Delta t} Z|^{p}\right) \leq 1+(1-\varepsilon) p\left[\alpha+\frac{1}{2}(p-1) \sigma^{2}\right] \Delta t
$$

Substituting this into (4.8) we obtain

$$
\lim _{k \rightarrow \infty} \frac{1}{k \Delta t} \log \mathbb{E}\left(\left|X_{k}\right|^{p}\right) \leq \frac{1}{\Delta t} \log \left(1+(1-\varepsilon) p\left[\alpha+\frac{1}{2}(p-1) \sigma^{2}\right] \Delta t\right) .
$$

But $\log (1+u) \leq u$ for $-1<u<0$, and thus (4.7) follows.

4.3. Exponential stability of the stochastic theta method. If we assume that $\Delta t$ is chosen so small that $\Delta t \alpha \theta<1$, then the ST method (2.3) applied to the linear SDE (4.1) may be written in the form

$$
X_{k+1}=X_{k}\left(1+\frac{\alpha}{1-\theta \alpha \Delta t} \Delta t+\frac{\sigma}{1-\theta \alpha \Delta t} \Delta B_{k}\right)
$$

This approximation coincides with the EM method applied to the modified linear SDE

$$
d y(t)=\frac{\alpha}{1-\theta \alpha \Delta t} y(t) d t+\frac{\sigma}{1-\theta \alpha \Delta t} y(t) d B(t) .
$$

Using this observation, it follows almost immediately that the statements of Theorems 4.2 and 4.3 also apply to the ST method.

5. Generalization to multidimensional nonlinear SDEs. To analyze the $n$ dimensional nonlinear SDE (2.1), we begin by imposing the linear growth assumption

$$
|f(x)| \vee|g(x)| \leq K|x| \quad \forall x \in \mathbb{R}^{n} .
$$

This implies

$$
f(0)=0, \quad g(0)=0,
$$

and we will be concerned with pathwise convergence of the solution $x(t)$ of $(2.1)$ to the zero solution, as $t \rightarrow \infty$, and the preservation of this property under discretization. We also note that condition (5.1) ensures that, with probability one, the solution will never reach the origin; see, for example, [15, Lemma 3.2].

We begin by giving sufficient conditions for almost sure exponential stability of the SDE.

THEOREM 5.1. Let (5.1) hold. If

$$
-\lambda:=\sup _{x \in \mathbb{R}^{n}, x \neq 0}\left(\frac{\langle x, f(x)\rangle+\frac{1}{2}|g(x)|^{2}}{|x|^{2}}-\frac{\langle x, g(x)\rangle^{2}}{|x|^{4}}\right)<0,
$$


then the solution of (2.1) obeys

$$
\limsup _{t \rightarrow \infty} \frac{1}{t} \log |x(t)| \leq-\lambda \quad \text { a.s. }
$$

and given any $\varepsilon \in(0, \lambda)$ there exists a $p^{\star} \in(0,1)$ such that for all $0<p<p^{\star}$

$$
\limsup _{t \rightarrow \infty} \frac{1}{t} \log \mathbb{E}\left(|x(t)|^{p}\right) \leq-p(\lambda-\varepsilon) .
$$

Proof. See Appendix A.

Next, we analyze the EM discretization (2.2).

TheOREM 5.2. Let (5.1) and (5.3) hold. Then for any $\varepsilon \in(0, \lambda)$ there is a constant $\Delta t^{\star} \in(0,1)$ such that for any $0<\Delta t<\Delta t^{\star}$ the EM approximation (2.2) satisfies

$$
\limsup _{k \rightarrow \infty} \frac{1}{k \Delta t} \log \left|X_{k}\right| \leq-(\lambda-\varepsilon) \quad \text { a.s. }
$$

Further, for any $\varepsilon \in(0, \lambda)$ and any sufficiently small $p>0$, there is a constant $\Delta t^{\star} \in(0,1)$ such that for any $0<\Delta t<\Delta t^{\star}$ the EM approximation (2.2) satisfies

$$
\limsup _{k \rightarrow \infty} \frac{1}{k \Delta t} \log \mathbb{E}\left(\left|X_{k}\right|^{p}\right) \leq-p(\lambda-\varepsilon)
$$

Proof. By condition (5.2), we compute from (2.2) that

$$
\begin{aligned}
\left|X_{k+1}\right|^{2} & =\left|X_{k}\right|^{2}+2\left\langle X_{k}, f\left(X_{k}\right) \Delta t+g\left(X_{k}\right) \Delta B_{k}\right\rangle+\left|f\left(X_{k}\right) \Delta t+g\left(X_{k}\right) \Delta B_{k}\right|^{2} \\
& =\left|X_{k}\right|^{2}\left(1+\xi_{k}\right),
\end{aligned}
$$

where

$$
\xi_{k}=\frac{1}{\left|X_{k}\right|^{2}}\left[2\left\langle X_{k}, f\left(X_{k}\right) \Delta t+g\left(X_{k}\right) \Delta B_{k}\right\rangle+\left|f\left(X_{k}\right) \Delta t+g\left(X_{k}\right) \Delta B_{k}\right|^{2}\right]
$$

if $X_{k} \neq 0$, otherwise it is set to -1 . Clearly, $\xi_{k} \geq-1$. For any $p \in(0,1)$, by inequality (4.9) we have

$$
\begin{aligned}
\left|X_{k+1}\right|^{p} & =\left|X_{k}\right|^{p}\left(1+\xi_{k}\right)^{p / 2} \\
& \leq\left|X_{k}\right|^{p}\left(1+\frac{p}{2} \xi_{k}+\frac{p(p-2)}{8} \xi_{k}^{2}+\frac{p(p-2)(p-4)}{2^{3} \times 3 !} \xi_{k}^{3}\right) .
\end{aligned}
$$

Hence the conditional expectation

$$
\begin{aligned}
\mathbb{E}\left(\left|X_{k+1}\right|^{p} \mid \mathcal{F}_{k \Delta t}\right) & \leq\left|X_{k}\right|^{p} \mathbb{E}\left(1+\frac{p}{2} \xi_{k}+\frac{p(p-2)}{8} \xi_{k}^{2}+\frac{p(p-2)(p-4)}{2^{3} \times 3 !} \xi_{k}^{3} \mid \mathcal{F}_{k \Delta t}\right) \\
& =\left|X_{k}\right|^{p} \mathbf{1}_{\left\{X_{k} \neq 0\right\}} \mathbb{E}\left(1+\frac{p}{2} \xi_{k}+\frac{p(p-2)}{8} \xi_{k}^{2}+\frac{p(p-2)(p-4)}{2^{3} \times 3 !} \xi_{k}^{3} \mid \mathcal{F}_{k \Delta t}\right),
\end{aligned}
$$

where $\mathbf{1}_{A}$ denotes the indicator function for $A$. Now,

$$
\begin{aligned}
& \mathbf{1}_{\left\{X_{k} \neq 0\right\}} \mathbb{E}\left(\xi_{k} \mid \mathcal{F}_{k \Delta t}\right) \\
= & 1_{\left\{X_{k} \neq 0\right\}} \mathbb{E}\left(\frac{1}{\left|X_{k}\right|^{2}}\left[2\left\langle X_{k}, f\left(X_{k}\right) \Delta t+g\left(X_{k}\right) \Delta B_{k}\right\rangle+\left|f\left(X_{k}\right) \Delta t+g\left(X_{k}\right) \Delta B_{k}\right|^{2}\right] \mid \mathcal{F}_{k \Delta t}\right) .
\end{aligned}
$$


Since $\Delta B_{k}$ is independent of $\mathcal{F}_{k \Delta t}$, we have $\mathbb{E}\left(\Delta B_{k} \mid \mathcal{F}_{k \Delta t}\right)=\mathbb{E}\left(\Delta B_{k}\right)=0$ and $\mathbb{E}\left(\left(\Delta B_{k}\right)^{2} \mid \mathcal{F}_{k \Delta t}\right)=\mathbb{E}\left(\left(\Delta B_{k}\right)^{2}\right)=\Delta t$. It is then easy to obtain that

$$
\begin{aligned}
\mathbf{1}_{\left\{X_{k} \neq 0\right\}} \mathbb{E}\left(\xi_{k} \mid \mathcal{F}_{k \Delta t}\right) & =\mathbf{1}_{\left\{X_{k} \neq 0\right\}}\left(\frac{1}{\left|X_{k}\right|^{2}}\left[2\left\langle X_{k}, f\left(X_{k}\right) \Delta t\right\rangle+\left|f\left(X_{k}\right)\right|^{2} \Delta t^{2}+\left|g\left(X_{k}\right)\right|^{2} \Delta t\right]\right) \\
& \leq \mathbf{1}_{\left\{X_{k} \neq 0\right\}}\left(\frac{1}{\left|X_{k}\right|^{2}}\left[2\left\langle X_{k}, f\left(X_{k}\right) \Delta t\right\rangle+\left|g\left(X_{k}\right)\right|^{2}\right] \Delta t+K^{2} \Delta t^{2}\right),
\end{aligned}
$$

where (5.1) has been used. Similarly, we can show that

$$
\mathbf{1}_{\left\{X_{k} \neq 0\right\}} \mathbb{E}\left(\xi_{k}^{2} \mid \mathcal{F}_{k \Delta t}\right) \geq \frac{4}{\left|X_{k}\right|^{4}}\left\langle X_{k}, g\left(X_{k}\right)\right\rangle^{2} \Delta t-c_{K} \Delta t^{2}
$$

and

$$
\mathbf{1}_{\left\{X_{k} \neq 0\right\}} \mathbb{E}\left(\xi_{k}^{3} \mid \mathcal{F}_{k \Delta t}\right) \leq c_{K} \Delta t^{2},
$$

where $c_{K}>0$ is a constant dependent only on $K$. Substituting (5.9), (5.10), and (5.11) into (5.8) and then using (5.3) and (5.1) we derive that

$$
\begin{aligned}
\mathbb{E}\left(\left|X_{k+1}\right|^{p} \mid \mathcal{F}_{k \Delta t}\right) \leq\left|X_{k}\right|^{p} \mathbf{1}_{\left\{X_{k} \neq 0\right\}}( & 1+\frac{p}{2\left|X_{k}\right|^{2}}\left[2\left\langle X_{k}, f\left(X_{k}\right) \Delta t\right\rangle+\left|g\left(X_{k}\right)\right|^{2}\right] \Delta t \\
& \left.+\frac{p(p-2)}{2\left|X_{k}\right|^{4}}\left\langle X_{k}, g\left(X_{k}\right)\right\rangle^{2} \Delta t+C \Delta t^{2}\right) \\
=\left|X_{k}\right|^{p} \mathbf{1}_{\left\{X_{k} \neq 0\right\}}\{ & 1+p \Delta t\left(\frac{\left\langle X_{k}, f\left(X_{k}\right)\right\rangle+\frac{1}{2}\left|g\left(X_{k}\right)\right|^{2}}{\left|X_{k}\right|^{2}}-\frac{\left\langle X_{k}, g\left(X_{k}\right)\right\rangle^{2}}{\left|X_{k}\right|^{4}}\right) \\
& \left.+\frac{p^{2} \Delta t\left\langle X_{k}, g\left(X_{k}\right)\right\rangle^{2}}{2\left|X_{k}\right|^{4}}+C \Delta t^{2}\right\} \\
\leq\left|X_{k}\right|^{p}(1-p \lambda \Delta & \left.+\frac{p^{2} \Delta t K^{2}}{2}+C \Delta t^{2}\right),
\end{aligned}
$$

where $C=C(K, p)>0$ is a constant independent of $\Delta t$. Now, for any given $\varepsilon \in(0, \lambda)$ and $p \in(0,1)$ sufficiently small for $p K^{2}<\varepsilon$, choose $\Delta t^{\star} \in(0,1)$ sufficiently small for $p \lambda \Delta t^{\star}<1$ and $C \Delta t^{\star}<\frac{1}{2} p \varepsilon$. It then follows from (5.12) that for any $\Delta t<\Delta t^{\star}$

$$
\mathbb{E}\left(\left|X_{k+1}\right|^{p} \mid \mathcal{F}_{k \Delta t}\right) \leq\left|X_{k}\right|^{p}(1-p(\lambda-\varepsilon) \Delta t) .
$$

Taking expectations on both sides yields

$$
\mathbb{E}\left(\left|X_{k+1}\right|^{p}\right) \leq \mathbb{E}\left(\left|X_{k}\right|^{p}\right)(1-p(\lambda-\varepsilon) \Delta t) .
$$

Since this holds for any $k \geq 0$, we have

$$
\mathbb{E}\left(\left|X_{k}\right|^{p}\right) \leq|x(0)|^{p}(1-p(\lambda-\varepsilon) \Delta t)^{k} \leq|x(0)|^{p} e^{-p k(\lambda-\varepsilon) \Delta t} \quad \forall k \geq 1 .
$$

This implies (5.7). Moreover, we have

$$
\mathbb{P}\left\{\left|X_{k}\right|^{p}>k^{2} e^{-p k(\lambda-\varepsilon) \Delta t}\right\} \leq \frac{|x(0)|^{p}}{k^{2}} \quad \forall k \geq 1 .
$$

By the Borel-Cantelli lemma, we see that for almost all $\omega \in \Omega$

$$
\left|X_{k}\right|^{p} \leq k^{2} e^{-p k(\lambda-\varepsilon) \Delta t}
$$


holds for all but finitely many $k$. Hence, there exists a $k_{0}(\omega)$, for all $\omega \in \Omega$ excluding a $\mathbb{P}$-null set, for which (5.14) holds whenever $k \geq k_{0}$. Consequently, for almost all $\omega \in \Omega$,

$$
\frac{1}{k \Delta t} \log \left|X_{k}\right| \leq-(\lambda-\varepsilon)+\frac{2 \log (k)}{p k \Delta t}
$$

whenever $k \geq k_{0}$. Letting $k \rightarrow \infty$ we obtain (5.6).

Let us now apply Theorem 5.2 to the linear SDE system

$$
d x(t)=A x(t) d t+G x(t) d B(t), \quad t \geq 0, \quad \text { given } 0 \neq x(0) \in \mathbb{R}^{n},
$$

where $A, G \in \mathbb{R}^{n \times n}$. This corresponds to $f(x)=A x$ and $g(x)=G x$ in (2.1). Note

$$
\frac{1}{2} \lambda_{\min }\left(A+A^{T}\right)|x|^{2} \leq\langle x, A x\rangle=\frac{1}{2}\left\langle x,\left(A+A^{T}\right) x\right\rangle \leq \frac{1}{2} \lambda_{\max }\left(A+A^{T}\right)|x|^{2}
$$

and

$$
\lambda_{\min }\left(G^{T} G\right)|x|^{2} \leq\left\langle x, G^{T} G x\right\rangle=|G x|^{2} \leq\|G\|^{2}|x|^{2},
$$

where $\lambda_{\max }(\cdot)$ and $\lambda_{\min }(\cdot)$ denote the maximum and minimum eigenvalues of a symmetric matrix, respectively. Moreover,

$$
0 \leq\langle x, G x\rangle^{2}=\frac{1}{4}\left\langle x,\left(G+G^{T}\right) x\right\rangle^{2} \leq \frac{1}{4} \lambda_{\max }^{2}\left(G+G^{T}\right)|x|^{2},
$$

while if $G+G^{T}$ is either nonpositive definite or nonnegative definite,

$$
\langle x, G x\rangle^{2} \geq \frac{1}{4}\left[\left|\lambda_{\max }\left(G+G^{T}\right)\right| \wedge\left|\lambda_{\min }\left(G+G^{T}\right)\right|\right]^{2}|x|^{4} .
$$

We hence observe that

$$
\begin{aligned}
\frac{\langle x, A x\rangle+\frac{1}{2}|G x|^{2}}{|x|^{2}}-\frac{\langle x, G x\rangle^{2}}{|x|^{4}} \geq & \frac{1}{2} \lambda_{\min }\left(A+A^{T}\right)+\frac{1}{2} \lambda_{\min }\left(G^{T} G\right) \\
& -\frac{1}{4} \lambda_{\max }^{2}\left(G+G^{T}\right),
\end{aligned}
$$

while if $G+G^{T}$ is either nonpositive definite or nonnegative definite,

$$
\begin{aligned}
\frac{\langle x, A x\rangle+\frac{1}{2}|G x|^{2}}{|x|^{2}}-\frac{\langle x, G x\rangle^{2}}{|x|^{4}} \leq & \frac{1}{2} \lambda_{\max }\left(A+A^{T}\right)+\frac{1}{2}\|G\|^{2} \\
& -\frac{1}{4}\left[\left|\lambda_{\max }\left(G+G^{T}\right)\right| \wedge\left|\lambda_{\min }\left(G+G^{T}\right)\right|\right]^{2} .
\end{aligned}
$$

By Theorem 5.2 we reach the following conclusion.

Corollary 5.3. If $G+G^{T}$ is either nonpositive definite or nonnegative definite and

$$
-\lambda:=\frac{1}{2} \lambda_{\max }\left(A+A^{T}\right)+\frac{1}{2}\|G\|^{2}-\frac{1}{4}\left[\left|\lambda_{\max }\left(G+G^{T}\right)\right| \wedge\left|\lambda_{\min }\left(G+G^{T}\right)\right|\right]^{2}<0,
$$

then for any $\varepsilon \in(0, \lambda)$ there is a pair of constants $p \in(0,1)$ and $\Delta t^{\star} \in(0,1)$ such that for any $\Delta t<\Delta t^{\star}$ the EM approximation of the linear $S D E$ (5.15) has the properties (5.7) and (5.6). 
6. Backward Euler. So far, we have proved positive results about EM for sufficiently small $\Delta t$. However, we saw in section 3 that this behavior does not extend to the cubic example (3.1). This SDE does not satisfy the linear growth condition (5.1); thus, of course, the theorems in section 5 do not apply. However, (3.1) does satisfy (5.3), since

$$
\sup _{x \in \mathbb{R}, x \neq 0}\left(\frac{\langle x, f(x)\rangle+\frac{1}{2}|g(x)|^{2}}{|x|^{2}}-\frac{\langle x, g(x)\rangle^{2}}{|x|^{4}}\right)=\sup _{x \in \mathbb{R}, x \neq 0}\left(\frac{x^{2}-x^{4}+2 x^{2}}{x^{2}}-\frac{4 x^{4}}{x^{4}}\right) \leq-1,
$$

and we note that the proof of Theorem 5.1 did not use the condition $|f(x)| \leq K|x|$ explicitly, though $|g(x)| \leq K|x|$ was used. Of course, the linear growth condition (5.1) was used implicitly to guarantee that the solution stays away from the origin with probability one. However, for this property we need only a weaker condition (see [15, Lemma 3.2 on p. 120]). Let us form this improved result as a new theorem.

THEOREM 6.1. The conclusions of Theorem 5.1 still hold if condition (5.1) is replaced by the following: for each integer $i \geq 1$ there is a $K_{i}>0$ such that

$$
|f(x)| \leq K_{i}|x| \quad \forall x \in \mathbb{R}^{n} \text { with }|x| \leq i,
$$

while there is a $K>0$ such that

$$
|g(x)| \leq K|x| \quad \forall x \in \mathbb{R}^{n} .
$$

An application of this theorem to the SDE (3.1) shows that its solution obeys (3.2), as claimed in section 3. We also saw from Lemma 3.1 that EM does not preserve this almost sure asymptotic stability for any $\Delta t>0$. Hence, it is not possible to extend Theorem 5.2 to the case where (5.1) is replaced by (6.1) and (6.2).

An interesting open question is whether any other numerical methods preserve exponential asymptotic stability for small $\Delta t$ under (6.1) and (6.2).

In this section we pursue a different approach. We consider a structural constraint that is known to allow positive results to be proved for the BE method in other contexts. More precisely, we assume that there is a constant $\mu \in \mathbb{R}$ such that

$$
\langle x-y, f(x)-f(y)\rangle \leq \mu|x-y|^{2} \quad \forall x, y \in \mathbb{R}^{n} .
$$

This one-sided Lipschitz condition has been applied in the deterministic and stochastic literature $[9,11,12,16,20]$ to establish results about long-term behavior and boundedness in a manner that is connected with the use of Lyapunov functions $[6,18]$. In particular, we note that under (6.3) the condition $\mu \Delta t<1$ ensures that (2.3) with $\theta=1$ can be solved uniquely for $X_{k+1}$.

The next theorem concerns the exponential stability of BE under conditions (6.3) and (6.2). Although (6.2) implies $g(0)=0,(6.3)$ may not force $f(0)=0$, and thus we still need to assume it for the purpose of stability analysis.

Theorem 6.2. Let (6.2) and (6.3) hold and $f(0)=0$. Assume also that $\mu+\frac{1}{2} \rho<$ 0 , where

$$
\rho:=\sup _{x \in \mathbb{R}^{n}, x \neq 0}\left(\frac{|g(x)|^{2}}{|x|^{2}}-\frac{2\langle x, g(x)\rangle^{2}}{|x|^{4}}\right) .
$$

Then (5.4) holds with $-\lambda=\mu+\frac{1}{2} \rho$, and for any $\varepsilon \in\left(0,\left|\mu+\frac{1}{2} \rho\right|\right)$ there is a pair of constants $p \in(0,1)$ and $\Delta t^{\star} \in(0,1)$ with $\mu \Delta t^{\star}<1$ such that for any $\Delta t<\Delta t^{\star}$, the 
BE method (that is, (2.3) with $\theta=1$ ) has the properties that

$$
\limsup _{k \rightarrow \infty} \frac{1}{k \Delta t} \log \mathbb{E}\left(\left|X_{k}\right|^{p}\right) \leq p\left(\mu+\frac{1}{2} \rho+\varepsilon\right)<0
$$

and

$$
\limsup _{k \rightarrow \infty} \frac{1}{k \Delta t} \log \left|X_{k}\right| \leq \mu+\frac{1}{2} \rho+\varepsilon<0 \quad \text { a.s. }
$$

Proof. It is straightforward to adapt the proof of Theorem 5.1 in order to establish (5.4) under (6.2) and (6.3). From (2.3) with $\theta=1$, we have

$$
\left|X_{k+1}\right|^{2}=\left\langle X_{k+1}, X_{k}+g\left(X_{k}\right) \Delta B_{k}\right\rangle+\left\langle X_{k+1}, f\left(X_{k+1}\right) \Delta t\right\rangle
$$

By (6.3) and $f(0)=0$, we have

$$
\left\langle X_{k+1}, f\left(X_{k+1}\right) \Delta t\right\rangle \leq \mu \Delta t\left|X_{k+1}\right|^{2} .
$$

But,

$$
\left\langle X_{k+1}, X_{k}+g\left(X_{k}\right) \Delta B_{k}\right\rangle \leq \frac{1}{2}\left|X_{k+1}\right|^{2}+\frac{1}{2}\left|X_{k}+g\left(X_{k}\right) \Delta B_{k}\right|^{2} .
$$

We hence obtain

$$
\begin{aligned}
\left|X_{k+1}\right|^{2} & \leq \frac{1}{1-2 \mu \Delta t}\left|X_{k}+g\left(X_{k}\right) \Delta B_{k}\right|^{2} \\
& \leq \frac{1}{1-2 \mu \Delta t}\left(\left|X_{k}\right|^{2}+2\left\langle X_{k}, g\left(X_{k}\right)\right\rangle \Delta B_{k}+\left|g\left(X_{k}\right)\right|^{2} \Delta B_{k}^{2}\right) \\
& =\frac{\left|X_{k}\right|^{2}}{1-2 \mu \Delta t}\left(1+\zeta_{k}\right)
\end{aligned}
$$

where

$$
\zeta_{k}=\frac{1}{\left|X_{k}\right|^{2}}\left(2\left\langle X_{k}, g\left(X_{k}\right)\right\rangle \Delta B_{k}+\left|g\left(X_{k}\right)\right|^{2} \Delta B_{k}^{2}\right)
$$

if $X_{k} \neq 0$, otherwise it is set to -1 . Clearly, $\zeta_{k} \geq-1$. For any $p \in(0,1)$, by inequality (4.9) we can then show that

$$
\begin{aligned}
\mathbb{E}\left(\left|X_{k+1}\right|^{p} \mid \mathcal{F}_{k \Delta t}\right) \leq \frac{\left|X_{k}\right|^{p}}{(1-2 \mu \Delta t)^{p / 2}} \mathbf{1}_{\left\{X_{k} \neq 0\right\}} \mathbb{E}\left(1+\frac{p}{2} \zeta_{k}\right. & \\
& \left.+\frac{p(p-2)}{8} \zeta_{k}^{2}+\frac{p(p-2)(p-4)}{2^{3} \times 3 !} \zeta_{k}^{3} \mid \mathcal{F}_{k \Delta t}\right) .
\end{aligned}
$$

In the same way as in the proof of Theorem 5.2 we can show that

$$
\begin{gathered}
\mathbf{1}_{\left\{X_{k} \neq 0\right\}} \mathbb{E}\left(\zeta_{k} \mid \mathcal{F}_{k \Delta t}\right)=\mathbf{1}_{\left\{X_{k} \neq 0\right\}} \frac{\left|g\left(X_{k}\right)\right|^{2}}{\left|X_{k}\right|^{2}} \Delta t, \\
\mathbf{1}_{\left\{X_{k} \neq 0\right\}} \mathbb{E}\left(\zeta_{k}^{2} \mid \mathcal{F}_{k \Delta t}\right) \geq \frac{4\left\langle X_{k}, g\left(X_{k}\right)\right\rangle^{2}}{\left|X_{k}\right|^{4}} \Delta t-K^{4} \Delta t^{2},
\end{gathered}
$$


and

$$
\mathbf{1}_{\left\{X_{k} \neq 0\right\}} \mathbb{E}\left(\zeta_{k}^{3} \mid \mathcal{F}_{k \Delta t}\right) \leq c_{K} \Delta t^{2}
$$

where $c_{K}>0$ is a constant dependent only on $K$. Substituting the three inequalities above into (6.7) and then using (6.4) and (6.2) we derive that

$$
\begin{aligned}
\mathbb{E}\left(\left|X_{k+1}\right|^{p} \mid \mathcal{F}_{k \Delta t}\right) \leq & \frac{\left|X_{k}\right|^{p}}{(1-2 \mu \Delta t)^{p / 2}} \mathbf{1}_{\left\{X_{k} \neq 0\right\}}\left(1+\frac{p}{2} \frac{\left|g\left(X_{k}\right)\right|^{2}}{\left|X_{k}\right|^{2}} \Delta t\right. \\
& \left.+\frac{p(p-2)}{8}\left[\frac{4\left\langle X_{k}, g\left(X_{k}\right)\right\rangle^{2}}{\left|X_{k}\right|^{4}} \Delta t-K^{4} \Delta t^{2}\right]+\frac{p(p-2)(p-4)}{2^{3} \times 3 !} c_{K} \Delta t^{2}\right) \\
\leq & \frac{\left|X_{k}\right|^{p}}{(1-2 \mu \Delta t)^{p / 2}}\left(1+\frac{1}{2} p \rho \Delta t+\frac{1}{2} p^{2} K^{2} \Delta t+C \Delta t^{2}\right),
\end{aligned}
$$

where $C=C(p, K)$ is a positive constant. Taking expectations on both sides, we arrive at

$$
\mathbb{E}\left(\left|X_{k+1}\right|^{p}\right) \leq \frac{1+\frac{1}{2} p \rho \Delta t+\frac{1}{2} p^{2} K^{2} \Delta t+C \Delta t^{2}}{(1-2 \mu \Delta t)^{p / 2}} \mathbb{E}\left(\left|X_{k}\right|^{p}\right)
$$

Now, for any $\varepsilon \in\left(0,\left|\mu+\frac{1}{2} \rho\right|\right)$, we may choose $p$ sufficiently small for $p K^{2} \leq \frac{1}{4} \varepsilon$. Then we have

$$
(1-2 \mu \Delta t)^{p / 2} \geq 1-p \mu \Delta t-\widehat{C} \Delta t^{2}>0,
$$

for sufficiently small $\Delta t$, where $\widehat{C}=\widehat{C}(p, \mu)$ is a positive constant. By further reducing $\Delta t$, if necessary, we may ensure that

$$
C \Delta t<\frac{1}{8} p \varepsilon, \quad \widehat{C} \Delta t<\frac{1}{4} \varepsilon, \quad\left|p\left(\mu+\frac{1}{4} \varepsilon\right) \Delta t\right| \leq \frac{1}{2} .
$$

Using (6.9) and (6.10) in (6.8) gives

$$
\mathbb{E}\left(\left|X_{k+1}\right|^{p}\right) \leq \frac{1+\frac{1}{2} p\left(\rho+\frac{1}{2} \varepsilon\right) \Delta t}{1-p\left(\mu+\frac{1}{4} \varepsilon\right) \Delta t} \mathbb{E}\left|X_{k}\right|^{p} .
$$

Note that for any $u \in\left[-\frac{1}{2}, \frac{1}{2}\right]$

$$
\frac{1}{1-u}=1+u+u^{2} \sum_{i=0}^{\infty} u^{i} \leq 1+u+u^{2} \sum_{i=0}^{\infty}\left(\frac{1}{2}\right)^{i}=1+u+2 u^{2} .
$$

By further reducing $\Delta t$, if necessary, so that

$$
4 p\left(\mu+\frac{1}{4} \varepsilon\right)^{2} \Delta t+\left(\rho+\frac{1}{2} \varepsilon\right)\left(p\left(\mu+\frac{1}{4} \varepsilon\right) \Delta t+2\left[p\left(\mu+\frac{1}{4} \varepsilon\right) \Delta t\right]^{2}\right) \leq \varepsilon,
$$

and using (6.11), we compute that

$$
\begin{aligned}
\mathbb{E}\left(\left|X_{k+1}\right|^{p}\right) & \leq\left(1+\frac{1}{2} p\left(\rho+\frac{1}{2} \varepsilon\right) \Delta t\right)\left(1+p\left(\mu+\frac{1}{4} \varepsilon\right) \Delta t+2\left[p\left(\mu+\frac{1}{4} \varepsilon\right) \Delta t\right]^{2}\right) \mathbb{E}\left(\left|X_{k}\right|^{p}\right) \\
& \leq\left[1+p\left(\mu+\frac{1}{2} \rho+\varepsilon\right) \Delta t\right] \mathbb{E}\left(\left|X_{k}\right|^{p}\right) .
\end{aligned}
$$

From this we can show the assertions (6.5) and (6.6) in the same way as in the proof of Theorem 5.2. 
Let us return to the scalar $\operatorname{SDE}(3.1)$, where $f(x)=x-x^{3}$ and $g(x)=2 x$. In this case, we have $\langle x-y, f(x)-f(y)\rangle \leq|x-y|^{2}$, so we may take $\mu=1$ in (6.3), while

$$
\rho:=\sup _{x \in \mathbb{R}, x \neq 0}\left(\frac{|g(x)|^{2}}{|x|^{2}}-\frac{2\langle x, g(x)\rangle^{2}}{|x|^{4}}\right)=-4,
$$

whence $\mu+\frac{1}{2} \rho=-1$, which gives another confirmation of (3.2).

By Theorem 6.2 , for any given $\varepsilon \in(0,1)$, there is a $\Delta t^{\star}>0$ sufficiently small so that if $\Delta t<\Delta t^{\star}$, the BE approximate solution of the SDE (3.1) obeys

$$
\limsup _{k \rightarrow \infty} \frac{1}{k \Delta t} \log \left|X_{k}\right| \leq-1+\varepsilon \quad \text { a.s. }
$$

which recovers property (3.2) very well indeed.

It is also interesting to observe that in the scalar case (that is, $n=1$ ),

$$
\rho=\sup _{x \in \mathbb{R}, x \neq 0}\left(-\frac{|g(x)|^{2}}{|x|^{2}}\right) \leq 0 .
$$

In this case, if (6.3) also holds with $\mu<0$, then the BE method is a.s. exponentially stable as long as the stepsize is sufficiently small. For example, the BE approximate solution to the scalar SDE

$$
d x(t)=\left(\mu x-x^{3}\right) d t+g(x) d B(t)
$$

is always a.s. exponentially stable as long as the stepsize is sufficiently small, $\mu<0$, and $g$ obeys the linear growth condition (6.2). However, in the case $\mu \geq 0$, we will need that

$$
|g(x)|^{2} \geq \rho x^{2}, \quad x \in \mathbb{R},
$$

holds for some $\rho>2 \mu$ in order to conclude that the BE method is a.s. exponentially stable.

7. Multidimensional noise. So far, in order to streamline the presentation, we have only considered scalar noise. In this section we state, without proof, how the nonlinear results generalize to the multinoise case, as follows:

$$
d x(t)=f(x(t)) d t+\sum_{j=1}^{d} g_{j}(x(t)) d B_{j}(t), \quad t \geq 0, \quad \text { given } 0 \neq x(0) \in \mathbb{R}^{n} .
$$

Here $\left(B_{1}(t), \ldots, B_{d}(t)\right)$ is a $d$-dimensional Brownian motion. As before, we assume, as a standing hypothesis, that $f, g_{1}, \ldots, g_{d}: \mathbb{R}^{n} \rightarrow \mathbb{R}^{n}$ are smooth enough for the SDE (7.1) to have a unique global solution $x(t)$ on $[0, \infty)$.

The following generalization of Theorem 6.1 gives a criterion for the almost sure and moment exponential stability of the SDE.

TheOrem 7.1. Assume that for each integer $i \geq 1$ there is a $K_{i}>0$ such that

$$
|f(x)| \leq K_{i}|x| \quad \forall x \in \mathbb{R}^{n} \text { with }|x| \leq i,
$$

while there is a $K>0$ such that

$$
\left|g_{j}(x)\right| \leq K|x| \quad \forall x \in \mathbb{R}^{n} \text { and } 1 \leq j \leq d .
$$


If

$$
-\lambda:=\sup _{x \in \mathbb{R}^{n}, x \neq 0}\left(\frac{\langle x, f(x)\rangle+\frac{1}{2} \sum_{j=1}^{d}\left|g_{j}(x)\right|^{2}}{|x|^{2}}-\frac{\sum_{j=1}^{d}\left\langle x, g_{j}(x)\right\rangle^{2}}{|x|^{4}}\right)<0,
$$

then the solution of (7.1) obeys

$$
\limsup _{t \rightarrow \infty} \frac{1}{t} \log |x(t)| \leq-\lambda \quad \text { a.s. }
$$

and given any $\varepsilon \in(0, \lambda)$ there exists a $p^{\star} \in(0,1)$ such that for all $0<p<p^{\star}$

$$
\limsup _{t \rightarrow \infty} \frac{1}{t} \log \mathbb{E}\left(|x(t)|^{p}\right) \leq-p(\lambda-\varepsilon)
$$

This theorem can be proved in a similar way that Theorem 5.1 is proved in the appendix.

The EM method applied to (7.1) produces approximations $X_{k} \approx x(k \Delta t)$ with $X_{0}=x(0)$ and

$$
X_{k+1}=X_{k}+\Delta t f\left(X_{k}\right)+\sum_{j=1}^{d} g\left(X_{k}\right) \Delta B_{j k}
$$

where $\Delta B_{j k}:=B_{j}((k+1) \Delta t)-B_{j}(k \Delta t)$. Recalling the motivating example in section 3 , we will replace the local linear growth condition (7.2) by a global one.

THEOREM 7.2. Assume that all the conditions of Theorem 7.1 hold with condition (7.2) replaced by

$$
|f(x)| \leq K|x| \quad \forall x \in \mathbb{R}^{n} .
$$

Then for any $\varepsilon \in(0, \lambda)$ there is a constant $\Delta t^{\star} \in(0,1)$ such that for any $0<\Delta t<\Delta t^{\star}$ the EM approximation (7.5) satisfies

$$
\limsup _{k \rightarrow \infty} \frac{1}{k \Delta t} \log \left|X_{k}\right| \leq-(\lambda-\varepsilon) \quad \text { a.s. }
$$

Further, for any $\varepsilon \in(0, \lambda)$ and any sufficiently small $p>0$, there is a constant $\Delta t^{\star} \in(0,1)$ such that for any $0<\Delta t<\Delta t^{\star}$ the EM approximation (7.5) satisfies

$$
\limsup _{k \rightarrow \infty} \frac{1}{k \Delta t} \log \mathbb{E}\left(\left|X_{k}\right|^{p}\right) \leq-p(\lambda-\varepsilon) .
$$

The BE method applied to (7.1) produces approximations $X_{k} \approx x(k \Delta t)$ with $X_{0}=x(0)$ and

$$
X_{k+1}=X_{k}+\Delta t f\left(X_{k+1}\right)+\sum_{j=1}^{d} g_{j}\left(X_{k}\right) \Delta B_{j k} .
$$

Theorem 7.3. Let (7.3) and (6.3) hold and $f(0)=0$. Assume also that $\mu+\frac{1}{2} \rho<$ 0 , where

$$
\rho:=\sup _{x \in \mathbb{R}^{n}, x \neq 0}\left(\frac{\sum_{j=1}^{d}\left|g_{j}(x)\right|^{2}}{|x|^{2}}-\frac{2 \sum_{j=1}^{d}\left\langle x, g_{j}(x)\right\rangle^{2}}{|x|^{4}}\right) .
$$


Then (7.4) holds with $-\lambda=\mu+\frac{1}{2} \rho$, and for any $\varepsilon \in\left(0,\left|\mu+\frac{1}{2} \rho\right|\right)$ there is a pair of constants $p \in(0,1)$ and $\Delta t^{\star} \in(0,1)$ with $\mu \Delta t^{\star}<1$ such that for any $\Delta t<\Delta t^{\star}$, the $B E$ method (7.6) has the properties that

$$
\limsup _{k \rightarrow \infty} \frac{1}{k \Delta t} \log \mathbb{E}\left(\left|X_{k}\right|^{p}\right) \leq p\left(\mu+\frac{1}{2} \rho+\varepsilon\right)<0
$$

and

$$
\limsup _{k \rightarrow \infty} \frac{1}{k \Delta t} \log \left|X_{k}\right| \leq \mu+\frac{1}{2} \rho+\varepsilon<0 \quad \text { a.s. }
$$

Theorems 7.2 and 7.3 can be proved in the same way as the scalar noise versions, Theorems 5.2 and 6.2 .

\section{Appendix A. Proof of Theorem 5.1.}

Proof. The result (5.4) may be proved by generalizing the analysis in [15, pp. 121-123], so we give only an outline. By the Itô formula, we can show that

$$
\begin{aligned}
\log \left(|x(t)|^{2}\right)= & \log \left(|x(0)|^{2}\right)+M(t) \\
& +\int_{0}^{t} 2\left(\frac{\langle x(s), f(x(s))\rangle+\frac{1}{2}|g(x(s))|^{2}}{|x(s)|^{2}}-\frac{\langle x(s), g(x(s))\rangle^{2}}{|x(s)|^{4}}\right) d s,
\end{aligned}
$$

where

$$
M(t)=\int_{0}^{t} \frac{2\langle x(s), g(x(s))\rangle}{|x(s)|^{2}} d B(s) .
$$

From the condition $|g(x)| \leq K|x|$, it is straightforward to show that

$$
\lim _{t \rightarrow \infty} \frac{M(t)}{t}=0 \quad \text { a.s. }
$$

Now, if (5.3) holds, then

$$
\log \left(|x(t)|^{2}\right) \leq \log \left(\left|x_{0}\right|^{2}\right)+M(t)-2 \lambda t .
$$

Dividing both sides by $2 t$ and then letting $t \rightarrow \infty$ we obtain (5.4).

Now we show (5.5). For $0<p<1$ we have, from the Itô formula,

$$
\begin{aligned}
d\left(|x(t)|^{p}\right)= & d\left(\left(|x(t)|^{2}\right)^{\frac{1}{2} p}\right) \\
= & \frac{p}{2}\left(|x(t)|^{2}\right)^{\frac{1}{2} p-1} d x(t) \\
& +\frac{1}{2} \frac{p}{2}\left(\frac{p}{2}-1\right)\left(|x(t)|^{2}\right)^{\frac{1}{2} p-2} 4\langle x(t), g(x(t))\rangle^{2} d t \\
= & p|x(t)|^{p}\left[\frac{\langle x(t), f(x(t))\rangle+\frac{1}{2}|g(x(t))|^{2}}{|x(t)|^{2}}-\frac{\langle x(t), g(x(t))\rangle^{2}}{|x(t)|^{4}}\right. \\
& \left.+\frac{p}{2} \frac{\langle x(t), g(x(t))\rangle^{2}}{|x(t)|^{4}}\right] d t \\
& +p|x(t)|^{p} \frac{\langle x(t), g(x(t))\rangle}{|x(t)|^{2}} d B(t) .
\end{aligned}
$$


Under (5.1) and (5.3) this implies

$$
\begin{aligned}
d\left(|x(t)|^{p}\right) \leq & p|x(t)|^{p}\left(-\lambda+\frac{p}{2} K^{2}\right) d t \\
& +p|x(t)|^{p} \frac{\langle x(t), g(x(t))\rangle}{|x(t)|^{2}} d B(t) .
\end{aligned}
$$

Given $\varepsilon \in(0, \lambda)$ we may choose $p \in(0,1)$ so small that $p K^{2} / 2<\varepsilon$, whence

$$
\begin{aligned}
d\left(e^{(\lambda-\varepsilon) p t}|x(t)|^{p}\right) \leq & e^{(\lambda-\varepsilon) p t}|x(t)|^{p}\left[(\lambda-\varepsilon) p+p\left(-\lambda+\frac{1}{2} p K^{2}\right)\right] d t \\
& +e^{(\lambda-\varepsilon) p t} p|x(t)|^{p} \frac{\langle x(t), g(x(t))\rangle}{|x(t)|^{2}} d B(t) \\
\leq & e^{(\lambda-\varepsilon) p t} p|x(t)|^{p} \frac{\langle x(t), g(x(t))\rangle}{|x(t)|^{2}} d B(t) .
\end{aligned}
$$

We deduce that

$$
e^{(\lambda-\varepsilon) p t} \mathbb{E}|x(t)|^{p} \leq \mathbb{E}|x(0)|^{p}
$$

and (5.5) follows.

\section{REFERENCES}

[1] J. A. D. Appleby, G. Berkolaiko, And A. Rodkina, On the Asymptotic Behavior of the Moments of Solutions of Stochastic Difference Equations, Technical report MS-05-21, School of Mathematical Sciences, Dublin City University, Dublin, Ireland, 2005.

[2] J. A. D. Appleby, D. Mackey, And A. Rodkina, Almost Sure Polynomial Asymptotic Stability of Stochastic Difference Equations, Technical report MS-05-20, School of Mathematical Sciences, Dublin City University, Dublin, Ireland, 2005.

[3] L. Arnold, Stochastic Differential Equations: Theory and Applications, Wiley, Chichester, UK, 1972.

[4] C. T. H. BAKER AND E. BUCKWAR, Exponential stability in p-th mean of solutions, and of convergent Euler-type solutions, of stochastic delay differential equations, J. Comput. Appl. Math., 184 (2005), pp. 404-427.

[5] A. BRyden And D. J. Higham, On the boundedness of asymptotic stability regions for the stochastic theta method, BIT, 43 (2003), pp. 1-6.

[6] E. Buckwar, R. Horvath-Bokor, And R. Winkler, Asymptotic mean-square stability of twostep methods for stochastic ordinary differential equations, BIT, 46 (2006), pp. 261-282.

[7] K. Burrage and T. Tian, A note on the stability properties of the Euler methods for solving stochastic differential equations, New Zealand J. Math., 29 (2000), pp. 115-127.

[8] P. M. Burrage, Runge-Kutta Methods for Stochastic Differential Equations, Ph.D. thesis, University of Queensland, Brisbane, Australia, 1999.

[9] E. Hairer And G. Wanner, Solving Ordinary Differential Equations II, Stiff and DifferentialAlgebraic Problems, 2nd ed., Springer-Verlag, Berlin, 1996.

[10] D. J. Higham, Mean-square and asymptotic stability of the stochastic theta method, SIAM J. Numer. Anal., 38 (2000), pp. 753-769.

[11] D. J. Higham And P. E. Kloeden, Numerical methods for nonlinear stochastic differential equations with jumps, Numer. Math., 101 (2005), pp. 101-119.

[12] D. J. Higham, X. MaO, And A. M. Stuart, Strong convergence of Euler-like methods for nonlinear stochastic differential equations, SIAM J. Numer. Anal., 40 (2002), pp. 10411063.

[13] X. Mao, Stability of Stochastic Differential Equations with Respect to Semimartingales, Pitman Res. Notes Math. Ser. 251, Longman Scientific and Technical, Harlow, UK, 1991.

[14] X. MAO, Exponential Stability of Stochastic Differential Equations, Marcel Dekker, New York, 1994.

[15] X. MAO, Stochastic Differential Equations and Applications, Horwood, Chichester, UK, 1997. 
[16] J. Mattingly, A. M. Stuart, and D. J. Higham, Ergodicity for SDEs and approximations: Locally Lipschitz vector fields and degenerate noise, Stochastic Process. Appl., 101 (2002), pp. 185-232.

[17] G. N. Milstein, E. Platen, and H. Schurz, Balanced implicit methods for stiff stochastic systems, SIAM J. Numer. Anal., 35 (1998), pp. 1010-1019.

[18] G. N. Milstein ANd M. V. Tretyakov, Numerical integration of stochastic differential equations with nonglobally Lipschitz coefficients, SIAM J. Numer. Anal., 43 (2005), pp. 11391154.

[19] Y. Saito And T. Mitsui, T-stability of numerical scheme for stochastic differential equations, in Contributions in Numerical Mathematics, World Sci. Ser. Appl. Anal. 2, World Scientific, River Edge, NJ, 1993, pp. 333-344.

[20] A. M. Stuart and A. R. Humphries, Dynamical Systems and Numerical Analysis, Cambridge University Press, Cambridge, UK, 1996. 